\title{
The Ubiquitous Pharmacogenomics consortium: making effective treatment optimization accessible to every European citizen
}

\author{
“...PREPARE trial will generate significant evidence showing the \\ positive effect of pre-emptive pharmacogenomics testing on lowering \\ clinically relevant adverse drug reactions...'
}

First draft submitted: 24 May 2017; Accepted for publication: 5 June 2017; Published online: 7 July 2017

Keywords: adverse drug reactions $\bullet$ block-randomized controlled trial $\bullet$ implementation - panel • pharmacogenomics • pre-emptive $\bullet$ PREPARE study $\bullet U-P G x$ consortium

Physicians and pharmacists increasingly recognize that pharmacogenomics (PGx)-informed prescribing and dispensing improves both the efficacy and safety of drug treatment $[1,2]$. An estimated $15 \%$ of the medicines recently evaluated by the EMA contain PGx information in the label that directly impacts patient treatment and this percentage will increase in the near future [3]. Several randomized controlled trials have shown the clinical utility of single drug-gene pre-emptive PGx testing. Examples include PGx testing to guide dosing of thiopurines, acenocoumarol and warfarin $[4,5,6]$, and PGx testing to guide drug selection for carbamazepine and abacavir $[7,8]$. Interestingly, this gold-standard evidence has focused on individual single gene-drug pair(s). Pre-emptive PGx testing for a panel of relevant pharmacogenes may be even more informative and clinically relevant than testing for individual gene-drug pairs. Reasons for this being threefold. First, PGx test results are static and can, therefore, be utilized lifelong. Second, over the course of their lifespan most patients will likely use multiple drugs of which multiple may benefit from PGx-informed prescribing [9]. Third, actionable PGx variants are highly common in the population and recent studies show that more than $95 \%$ of the population carry at least one actionable PGx variant when tested for a panel of six to eight well-recognized pharmacogenes $[10,11,12,13]$. Indeed, the positive effects of pre-emptive PGx testing for a panel of genes have recently been demonstrated in several small studies $[14,15,16]$. Nevertheless, convincing evidence from well-designed, sufficiently powered prospective studies is needed. To fill this unmet need and to achieve implementation of PGxguided prescribing in Europe, the Ubiquitous Pharmacogenomics (U-PGx) consortium was founded.

\section{U-PGx consortium}

The U-PGx consortium is a collaborative effort of experts across 16 different organizations in ten European countries established to address the remaining challenges and obstacles for implementation of PGx-informed prescribing into patient care [17]. The U-PGx consortium is funded by a 15 million euro Horizon 2020 grant from the European Commission (grant no. 668353). The project started in January 2016 and will run for 5 years. The U-PGx consortium aims to make effective treatment optimization accessible in all of Europe. To achieve this, the U-PGx consortium is conducting a prospective, block-randomized controlled clinical trial across multiple genes, multiple drugs, multiple ethnicities and multiple healthcare systems called PREPARE (PREemptive Pharmacogenomic testing for preventing Adverse drug REactions [ADRs]) [18].
Lisanne EN Manson', Cathelijne $\mathrm{H}$ van der Wouden', Jesse J Swen*,1 \& Henk-Jan Guchelaar ${ }^{1}$ ${ }^{1}$ Department of Clinical Pharmacy \& Toxicology, Leiden Network for Personalised Therapeutics, Leiden University Medical Centre, 2300 RC Leiden, The Netherlands *Author for correspondence: Tel.: +310715262790 j.j.swen@lumc.nl 
The U-PGx consortium consists of four different components (Figure 1). The first component has developed the enabling tools necessary to implement PGx testing and integrate PGx test results into the different electronic health records and clinical decision support systems (CDSS) across Europe. The developed tools include the selection of a genotyping platform and the selection of a panel encompassing 50 variants to be tested covering 13 pharmacogenes. A comprehensive educational program about $\mathrm{PGx}$, including e-learning modules available from the U-PGx website [17], was created and eight U-PGx personalized medicine days will be organized to educate healthcare professionals. To educate the general public, two public events will be organized, of which the first will be held in London in December 2017 [17].

"A 'Safety Code Card' has been developed to make pharmacogenomics data and clinical decision support systems available in countries and healthcare systems in which electronic health record is not available."

Essential for the project is the translation of more than 80 guidelines available from the Dutch
Pharmacogenomics Working Group guidelines into local languages (Greek, Slovenian, English, German, Spanish and Italian). These guidelines are based upon systematic review of literature and can be integrated as CDSS in electronic prescribing systems and medication surveillance systems [19]. A 'Safety Code Card' has been developed to make PGx data and CDSS available in countries and healthcare systems in which electronic health record is not available. This patient-specific card contains a QR code, which can be scanned and will lead the medical professional directly to a website that provides recommendations customized to the PGx profile of that specific patient (Figure 2). The work of the first component paved the way for the second component, which contains the actual clinical trial (PREPARE). The third component will focus on expanding our understanding of the influence of genetic variation on drug response with a specific focus on the contribution of rare $(<1 \%)$ variants and extreme phenotypes. Second, this component aims to integrate our knowledge of drug-drug and gene-drug interactions. The fourth component focuses on ethical and legal issues surrounding the U-PGx project.

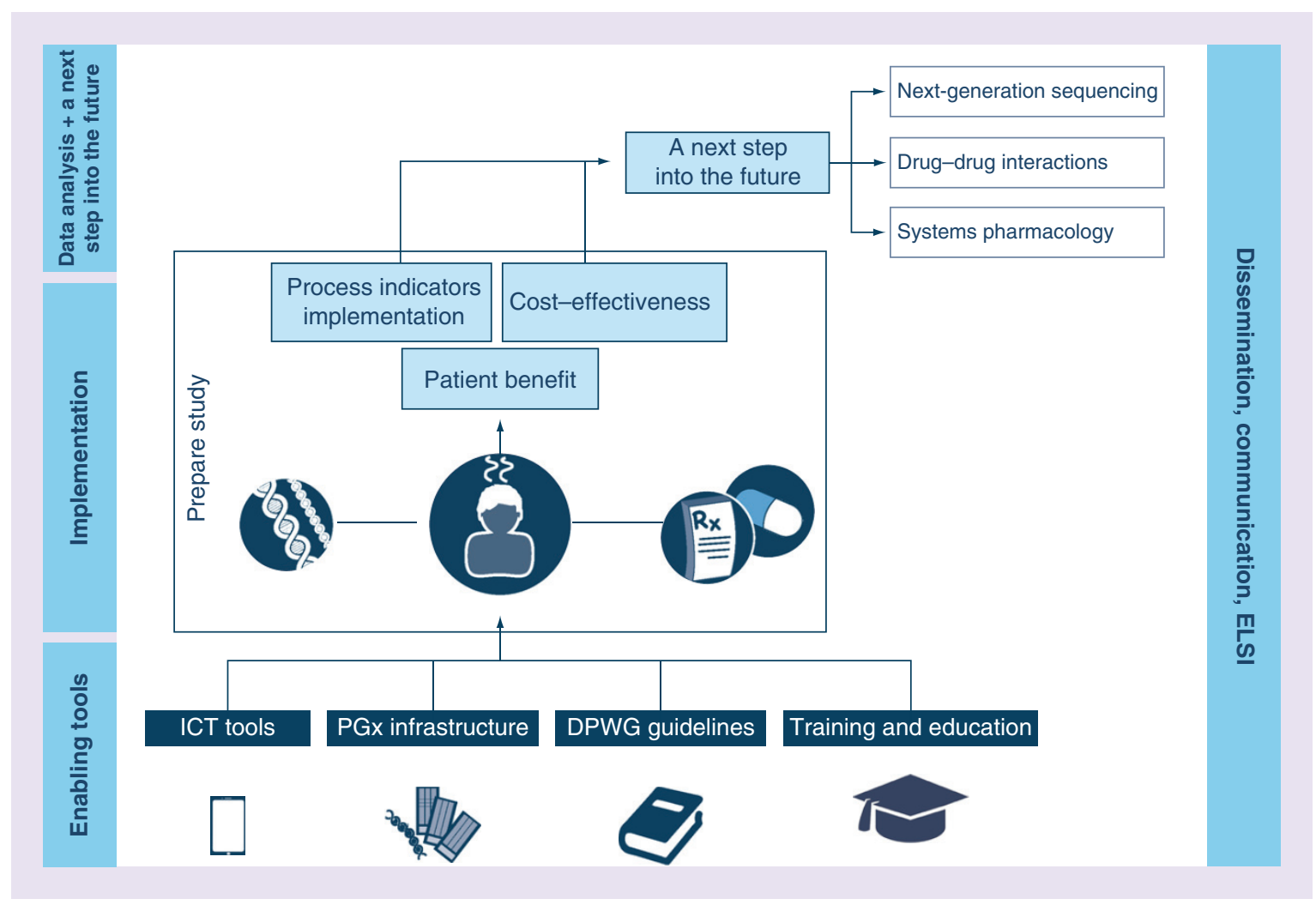

Figure 1. An overview of the Ubiquitous Pharmacogenomics project. Enabling tools have been developed to pave the way for the implementation of PGx guided prescribing in the PREPARE study. The PREPARE study will generate data that can be used in innovative projects with the aim to expand our understanding of PGx. The final component focuses on any legal and ethical issues.

DPWG: Dutch Pharmacogenomics Working Group; ELSI: Ethical, legal, and societal impact; ICT: Information and communication technology; PGx: Pharmacogenomics. 


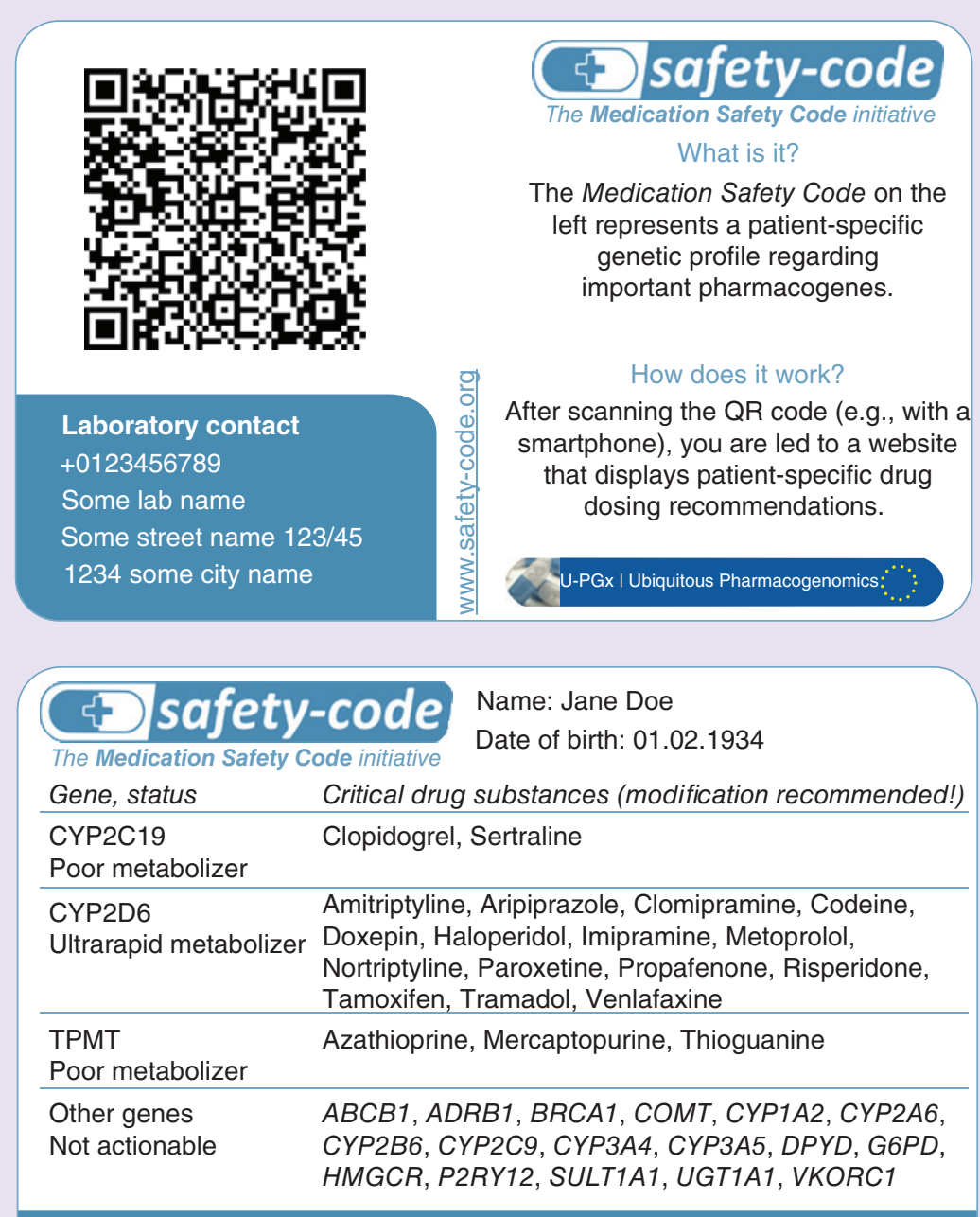

Figure 2. The Safety Code Card contains pharmacogenetic information and a QR code, which can be scanned and will lead the medical professional directly to a website that provides recommendations customized to the PGx profile of that specific patient.

PGx: Pharmacogenomics.

Data taken from [20].

\section{PREPARE study}

The PREPARE study is unique in its multigene, multidrug, multicenter, multi-ethnic and multihealthcare system approach. PREPARE will implement pre-emptive genotyping of a panel of 13 pharmacogenes consisting of 50 variants into routine care to guide drug and dose selection for 41 drugs for which Dutch Pharmacogenomics Working Group guidelines exist. This will be achieved by means of an international, prospective, block-randomized controlled study $(\mathrm{n}=8100)$ across institutes in seven European countries (The Netherlands, UK, Greece, Italy, Slovenia, Spain and Austria). The settings differ between the countries, for example, The Netherlands recruits primary care patients through community pharmacies while Italy recruits cancer patients through oncology departments and Spain recruits patients through a cardiology department. The PREPARE study will run for 36 months and is split into two 18 months blocks.

"...unique in its multigene, multidrug,

multicenter, multi-ethnic and multihealthcare system approach."

The participating countries are block-randomized to start with either standard of care or implementing PGxguided prescribing. After 18 months, the countries will switch to the opposite strategy and will recruit new patients, thus serving as their own control group and thereby balancing any existing differences in standard of care between centers in the seven countries [18]. 
All patients will have a follow-up of a minimum of 12 weeks and a maximum of 18 months. During this follow-up all adverse events within 12 weeks of starting the index drug (one of the 41 study drugs for which the patient is included) will be collected using results from online questionnaires (at 2 and 8 weeks) and from telephone calls (at 4 and 12 weeks and at the end of the study arm). It is expected about $50 \%$ of the patients will receive a second and $30 \%$ will even receive a third prescription of a study drug within the PREPARE study. This will initiate an identical follow-up as for the initial drug of inclusion and will result in more information on the effects of pre-emptive PGx testing [18].

PREPARE aims to evaluate the impact of implementation of PGx guided prescribing on clinical outcomes and its cost-effectiveness. The primary endpoint is the occurrence of at least one ADR within 12 weeks of initiating the index drug, which contributes to the primary composite endpoint. We expect to find a decrease of clinically relevant ADRs of approximately 30\% in the intervention arm compared with the standard of care arm. Secondary outcomes include the number of drug cessations, the number of drug changes and the number of serious ADRs, which are all expected to fall. Furthermore, process indicators for implementation will be collected to assess how PGx-guided prescribing impacts individual patient care and care on a nationwide level. Moreover, data on costs associated with ADRs will be collected to perform a country-specific cost-effectiveness analysis.

The PREPARE trial has been approved by ethics committees in all seven countries and has been registered on clinicaltrials.gov (NCT03093818). The trial is open and the sites are recruiting.

\section{A next step into the future}

Our understanding of how pharmacogenetic variation affects drug response is rapidly evolving. However, even after accounting for our current pharmacogenetic knowledge many unexplained drug responses remain. It was recently suggested that low frequency $(<1 \%)$ variants significantly impact drug pharmacokinetics [21]. Therefore, advancing our knowledge of PGx may significantly increase the benefits of PGxinformed prescribing in the future. The U-PGx consortium hopes to achieve this in two ways. First, by

\section{References}

1 Bank PC, Swen JJ, Guchelaar HJ. A nationwide survey of pharmacists' perception of pharmacogenetics in the context of a clinical decision support system containing pharmacogenetics dosing recommendations. Pharmacogenomics 18(3), 215-225 (2017). performing a follow-up study among patients with extreme phenotypes within the PREPARE study. Next generation sequencing will be applied to identify rare, unknown variants associated with drug response, and blood plasma levels of the drug of interest and its relevant metabolites within $24 \mathrm{~h}$ of a serious ADR will be measured to confirm extreme phenotypes. Second, patients can participate in a pharmacokinetic sub-study by providing additional blood samples for a number of drugs. Through a systems pharmacology approach, nongenetic determinants of drug response such as age, disease-related factors and drug-drug interactions are integrated to create novel and better models of personalized medicine. By doing this research, the U-PGx consortium hopes to contribute to unraveling the gaps in understanding the genetic impact on drug responses.

\section{PGx-informed prescribing in all of Europe}

With the conception and funding of the U-PGx consortium, the EU has made substantial progress in the implementation of PGx in daily clinical care. The U-PGx consortium hypothesizes that the PREPARE trial will generate significant evidence showing the positive effect of pre-emptive PGx testing on lowering clinically relevant ADRs through its multicenter, multidrug, multigene, multi-ethnic and multihealthcare approach. By conducting the PREPARE study the U-PGx consortium also aims to demonstrate costeffectiveness of a pre-emptive PGx screening approach. In the process, experience in implementing PGx testing in the standard of care workflow is gained within different settings in multiple countries in Europe, paving the way for implementation throughout all of Europe and making pre-emptive PGx testing in Europe a reality in the near future.

Financial \& competing interests disclosure

This work received funding from the European Community's Horizon 2020 Programme under grant agreement no. 668353 (Ubiquitous Pharmacogenomics [U-PGx]). The authors have no other relevant affiliations or financial involvement with any organization or entity with a financial interest in or financial conflict with the subject matter or materials discussed in the manuscript apart from those disclosed.

No writing assistance was utilized in the production of this manuscript.

2 Stanek EJ, Sanders CL, Taber KA et al. Adoption of pharmacogenomic testing by US physicians: results of a nationwide survey. Clin. Pharmacol. Ther. 91(3), 450-458 (2012).

3 Ehmann F, Caneva L, Prasad K et al. Pharmacogenomic information in drug labels: European Medicines Agency perspective. Pharmacogenomics J. 15(3), 201-210 (2015). 
4 Cerezo-Manchado JJ, Roldan V, Corral J et al. Genotypeguided therapy improves initial acenocoumarol dosing. Results from a prospective randomised study. Thromb. Haemost. 115(1), 117-125 (2016).

5 Pirmohamed M, Burnside G, Eriksson N et al. A randomized trial of genotype-guided dosing of warfarin. N. Engl. J. Med. 369(24), 2294-2303 (2013).

6 Coenen MJ, de Jong DJ, van Marrewijk CJ et al. Identification of patients with variants in TPMT and dose reduction reduces hematologic events during thiopurine treatment of inflammatory bowel disease. Gastroenterology 149(4), 907.e7-917.e7 (2015).

7 Chen P, Lin JJ, Lu CS et al. Carbamazepine-induced toxic effects and $H L A-B^{*} 1502$ screening in Taiwan. N. Engl. J. Med. 364(12), 1126-1133 (2011).

$8 \quad$ Mallal S, Phillips E, Carosi G et al. HLA-B*5701 screening for hypersensitivity to abacavir. N. Engl. J. Med. 358(6), 568-579 (2008).

9 Samwald M, Xu H, Blagec K et al. Incidence of exposure of patients in the United States to multiple drugs for which pharmacogenomic guidelines are available. PLoS ONE 11(10), e0164972 (2016).

10 Dunnenberger HM, Crews KR, Hoffman JM et al. Preemptive clinical pharmacogenetics implementation: current programs in five US medical centers. Annu. Rev. Pharmacol. Toxicol. 55, 89-106 (2015).

11 Van Driest SL, Shi Y, Bowton EA et al. Clinically actionable genotypes among 10,000 patients with preemptive pharmacogenomic testing. Clin. Pharmacol. Ther. 95(4), 423-431 (2014).

12 Goh LL, Lim CW, Sim WC, Toh LX, Leong KP. Analysis of genetic variation in CYP450 genes for clinical implementation. PLoS ONE 12(1), e0169233 (2017).
13 Bush WS, Crosslin DR, Owusu-Obeng A et al. Genetic variation among 82 pharmacogenes: the PGRNseq data from the eMERGE network. Clin. Pharmacol. Ther. 100(2), 160-169 (2016).

14 Elliott LS, Henderson JC, Neradilek MB, Moyer NA, Ashcraft KC, Thirumaran RK. Clinical impact of pharmacogenetic profiling with a clinical decision support tool in polypharmacy home health patients: a prospective pilot randomized controlled trial. PLoS ONE 12(2), e0170905 (2017).

15 Finkelstein J, Friedman C, Hripcsak G, Cabrera M. Potential utility of precision medicine for older adults with polypharmacy: a case series study. Pharmgenomics Pers. Med. 9, 31-45 (2016).

16 Brixner D, Biltaji E, Bress A et al. The effect of pharmacogenetic profiling with a clinical decision support tool on healthcare resource utilization and estimated costs in the elderly exposed to polypharmacy. J. Med. Econ. 19(3), 213-228 (2016).

17 Ubiquitous Pharmacogenomics C. www.U-PGx.eu

18 van der Wouden $\mathrm{CH}$, Cambon-Thomsen A, Cecchin E et al. Implementing pharmacogenomics in Europe: design and implementation strategy of the Ubiquitous Pharmacogenomics consortium. Clin. Pharmacol. Ther. 101(3), 341-358 (2017).

19 Swen JJ, Nijenhuis M, de Boer A et al. Pharmacogenetics: from bench to byte--an update of guidelines. Clin. Pharmacol. Ther. 89(5), 662-673 (2011).

20 Safety-code. http://safety-code.org/

21 Kozyra M, Ingelman-Sundberg M, Lauschke VM. Rare genetic variants in cellular transporters, metabolic enzymes, and nuclear receptors can be important determinants of interindividual differences in drug response. Genet. Med. 19(1), 20-29 (2017). 